\title{
An Architecture for Bidirectional Learning Games
}

\author{
Tanja von Leipzig, Stellenbosch University, South Africa* \\ Eric Lutters, University of Twente, The Netherlands \\ (iD https://orcid.org/0000-0001-7694-8453 \\ Vera Hummel, Reutlingen University, Germany \\ Corné Schutte, Stellenbosch University, South Africa
}

\begin{abstract}
Dynamic personalization of learning trajectories that integrate different perspectives and variable scenarios is a viable way to improve the effectiveness and efficiency of training and education. Serious games offer a designated platform for this by aggregating learner interactions and using these to dynamically configure, adjust, and tailor the game to individuals and contexts. An architecture is presented to support the creation of serious games for specific scenarios in a faster, more effective, and efficient manner. Following a research-by-design approach, the architecture is simultaneously developed and applied in case studies, with the experiences infused as enhancements for subsequent design iterations.
\end{abstract}

\section{KEYWORDS}

Adaptive Serious Games, Architecture, Bidirectional Learning, Effective Game Design, Efficient Game Design, Game-Based Learning, Research-by-Design, Stakeholder Co-Design

\section{INTRODUCTION}

The potential of game-based learning (GBL) to provide effective learning environments is widely recognized (Connoly, Boyle, MacArthur, Hainey, \& Boyle, 2012; Hamari, et al., 2016; Monterrat, Desmarais, Lavoué, \& George, 2015). Codish and Ravid (2014) define GBL as the use of games with an educational objective. Serious games, games developed with a primary purpose other than entertainment, such as education or training, is a term often used interchangeably with GBL. Whilst the focus of GBL to date has been on improved learning outcomes of learners, few consider the impact of gameplay on other stakeholders. Stakeholders may include a combination of the learner, the education provider and facilitator, the sponsor of the learning or training instance, the real-world system or environment portrayed, and indirect stakeholders specific to the scenario domain that are impacted by gameplay. Even fewer instances consider the dynamic personalization of learning trajectories by integrating different perspectives and variable scenarios. Serious games offer a potential platform to aggregate learner behaviors and results, and use these to dynamically configure, adjust and tailor the 
game to individuals and contexts, ultimately providing a learning environment of improved quality, effectiveness and efficiency.

An architecture is presented to support the creation of serious games for specific scenarios, in a faster, more effective and more efficient manner. Through its modular structure and emphasis on reusability and configuration of existing tools and functionalities, the architecture enables the creation of serious games in a faster and more efficient manner. Mechanisms such as parameterized learning and contextualized adaptation of content in response to learner behaviors and results, increase the effectiveness of the game design. The architecture emphasizes the use of games to provide learning and training for various stakeholders of the game, not only the learner/end-user. Furthermore, the aggregation of behaviors and results to further improve the game mechanics, learning material and approach, as well as various other parameters that may influence the learning taking place are incorporated in the architecture. The primary research question addressed is two-fold. Firstly, whether such an architecture will enable the faster, more effective, and more efficient creation of serious games for specific scenarios. Secondly, whether the resulting games provide effective and efficient learning opportunities for multiple stakeholders.

The architecture is developed in such a way that serious games can be created in a faster, more effective, more efficient manner the more it is used, through mechanisms such as modularity and reusability. To this end, the development and validation of the architecture is attached to a number of case studies. Here, the focus lies on a case study in the diagnostic laboratory environment to demonstrate the usability of the architecture. More specifically, the focus of the case study is a series of courses aimed at conveying process understanding and teaching performance improvement to diagnostic laboratory professionals. In order to provide customers with more flexibility in completing the originally classroom-based training, the courses have been adapted for online consumption. The training provider has recognized the potential of technology, and especially games, in addressing some of the limitations of, as well as enhancing and even surpassing conventional methods of training.

\section{Game-Based Learning}

Games are built on intrinsic motivation, in which challenge and skill are balanced to be "pleasantly frustrating" (Hall, Watson, \& Kitching, 2017, p. 6). Gee (2003) believes this inherent intrinsic motivation of games to be the reason that they are the future of learning. Games here are considered to be playable games in which narratives, role-play, game elements and mechanisms are effectively combined with didactical content and learning paths to provide an immersive, effective learning environment. It excludes lower-level learning games such as games focusing primarily on memorization or reciting of theory.

Games' motivational power can be attributed to their dynamic, responsive, visualized nature, which, combined with elements such as novelty, focused goals, variation, choice and affirmation of performance, provide favorable learning experiences (Dickey, 2005).

\section{Potential Benefits of Game-Based Learning}

Amory and Seagram (2003) discuss the positive effect of games on cognitive function and motivation, by inherently stimulating curiosity. Furthermore, games promote goal formation, competition, intrinsic motivation and self-evaluation, and provide an environment in which the player is both behaviorally and metacognitively active. Gameplay requires a skillset synonymous to quality learning, including logic, critical thinking, memory, visualization and problem-solving abilities. Quality learning environments stimulate the effective development of fundamental skills by actively engaging the learner in educational content and scenarios (Amory \& Seagram, 2003; Romero, Usart, \& Ott, 2015).

Furthermore, serious games provide a safe opportunity for players to learn and experiment in realistic environments (van der Vegt, Westera, Nyamsuren, Georgiev, \& Ortiz, 2016). They stimulate problem ownership through roleplay and support the acquisition of both tacit and contextualized knowledge by promoting a learn-by-doing approach to learning. 
Oblinger (2004, p. 14) draws clear parallels between pedagogical best practices and conditions exhibited in game environments:

- Individualization: Adaptation to the individual's preferences and level.

- Feedback: Immediate and contextualized feedback.

- Active learning: Active environment leading to discovery.

- Motivation: Hours of engagement in pursuit of a goal.

- Social: Playable with others (e.g., multiplayer games) or involve communities of users.

- Scaffolding: Players cannot move to higher levels until competence is displayed at the current level.

- Transfer: Enables transfer of information from an existing context to a novel one.

- Assessment: Evaluation of skill and comparison to others.

The characteristics and benefits of games demonstrate that they provide a suitable learning environment and can lead to successful learning above and beyond what can be achieved in traditional learning environments. The ability to adapt to individual preferences and abilities combined with appropriate embedding enables efficient learning, whereby learning goals can be achieved with the minimum required resources and/or effort. By promoting active learning and targeting specific content and desired learning outcomes, serious games cater for more effective learning. Moreover, they cultivate problem-solving and relevant work skills, which, combined with transfer, has the potential to improve the quality of learning. Given all the potential benefits, adoption of games in learning has still been slow.

\section{Shortcomings and Limitations of Current Games in Learning}

Despite the increasing interest in serious games, the uptake thereof in industry and education settings has been limited. Organizations are often still unconvinced of the benefits of serious games for corporate training and education and have a generally risk-averse attitude toward new technologies and/or the perceived development costs (Carvalho, 2016). Most serious games are conceived as one-of-a-kind products, tailored to the client's requirements and as such, have a very low reusability of both the final product and its individual components (Carvalho, 2016; van der Vegt, Westera, Nyamsuren, Georgiev, \& Ortiz, 2016).

In addition, the games often fail to take into account the individual differences in end-users (Codish \& Ravid, 2014; Molin, 2017; Monterrat, Desmarais, Lavoué, \& George, 2015; Shi, Christea, $\&$ Hadzidedic, 2014). Not only are different learners motivated by different elements, but they also respond best to different forms of information (Cilliers, 2016; Minović \& Milovanović, 2013) and approach and play a game differently (Molin, 2017). For GBL to be effective, a highly personalized perspective should be taken in which individual differences are considered and the contents and mechanisms adapted accordingly (Akbulut \& Cardak, 2012; All, Castellar, \& Van Looy, 2016; Ferro, Walz, \& Greuter, 2013; Gofron, 2014; Göbel, Wendel, Ritter, \& Steinmetz, 2010).

Developing serious games of a sufficient quality to enable effective and efficient learning, is a complex and challenging endeavor. Appropriately balancing pedagogical practices and the game aspects and mechanics to realize those, is challenging (Carvalho, 2016; van der Vegt, Westera, Nyamsuren, Georgiev, \& Ortiz, 2016). Furthermore, the development lead-time and investment are often high.

Finally, serious games are usually developed as once-off products and thus have a low resilience to aging. They may quickly become outdated if or when new tools, methods and technologies become available. To be effective in the long-term, serious games should be designed in such a way that they can be updated or adapted if needed. Carvalho (2016) emphasizes the importance of having an effective model guiding the design and development aspects of serious game development. 


\section{Existing Game Design Models and their Limitations}

There are various existing models for the design of serious games, including models and frameworks for the design of adaptive serious games. Examples include the six facets of serious learning game design by Marne et al. (2012), Wright's (2015) conceptual model for personalized learning, and the personalized gamification model proposed by González et al (2016). A review of existing design models led to the identification of various limitations, including that in many instances, the proposed models have not been practically tested. Moreover, most only consider individual aspects as opposed to the full complexity of the learning process and game design aspects. Although some models include aspects of personalization or adaptation, it was found that personalization or once-off adaptation was insufficient, and that dynamic adaptation is required for more effective and efficient serious games.

\section{An Initial Framework Proposed For Personalized, Adaptive Games}

In an attempt to address the above-mentioned limitations, a framework for personalized, dynamically adaptive serious games was presented by the authors in a related work (von Leipzig, von Leipzig, \& Hummel, 2019). The framework consists of 5 stages that are iteratively executed, namely personalized game design, development, gameplay assessment and adaptation, an adjustment period and artefact assessment. The first stage (Figure 1[REMOVED REF FIELD]) focuses on game design, from domain simulation through to assessment and design iterations. In the second stage, the game is developed and tested, with any feedback fed back into the design. Three dynamic events are included in the gameplay stage, namely the assessment of learning, the assessment for learning and the adaptation of content. Again, any feedback is fed back into the design stage. An adjustment period is introduced, before the game's effectiveness is evaluated in the final stage, with feedback fed back into the design of the next iteration.

The framework was validated through expert interviews as well as its application in a first case study. Six experts from the various disciplines of pedagogy, game design, data and analytics, game development, and adaptation were interviewed to evaluate the relevance and validity of the framework. Overall, the responses were positive with $100 \%$ of the experts stating that the framework is relevant and can be used to design and develop effective games. The framework was subsequently applied in a first case study, where a game was designed and developed for an engineering economics module at Stellenbosch University. A diverse group of 14 students were asked to play the game as beta testers. Various interactions and behaviors were tracked during gameplay and analyzed to identify any patterns and insights. Pre- and post-test quizzes were implemented to evaluate the level of learning through the game. Furthermore, each player was asked to complete a survey relating to their overall impression and feedback. The results were largely positive, with $100 \%$ of players stating that they had learnt effectively through the game and would enjoy playing more such games as part of the curriculum. The analysis of the pre- and post-test answers also reflected an improvement in understanding by all players, with the most significant improvement seen in the players with the lowest initial scores. From the feedback obtained through the expert interviews, and an analysis of the case study results and player behaviors, various lessons and unexploited benefits were identified.

\section{Lessons and Unexploited Benefits}

One of the main opportunities identified in the framework validation was the potential of bidirectional learning through targeted game environments. Bidirectional learning, where learning simultaneously takes place in a two-way direction (Zhang, Wang, \& Yun, 2015), has great potential and has, to date, not been incorporated in serious game design. Whilst many studies have focused on the learning via serious games from an end-user perspective, none have been found that consider learning from a systems perspective. If a serious game portrays a real-world environment, analyzing and aggregating learner behavior and results may help identify improvements in the real-world environment (i.e., the system learns based on learner interactions and choices). Bidirectional learning could also prove 


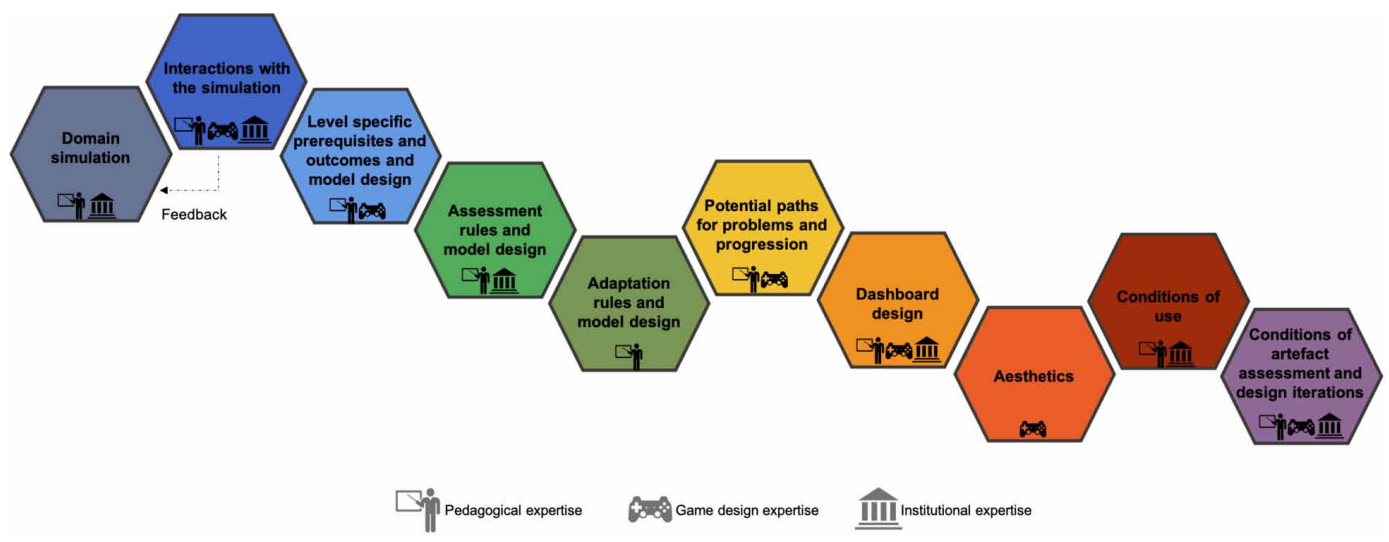

valuable to the education/training provider since the aggregated behavior and results may indicate content-related improvement opportunities.

Furthermore, Kalyanakrishnan and Stone (2011) propose the use of parameterized learning to enable more effective learning environments, in which parameters are systematically controlled and adjusted according to the specific scenario and desired learning outcomes. Whilst the developed framework already aims to adapt the game and its contents to individual users, bidirectional learning would enable the contextualized adaptation of learning material, experiences and learning trajectories based on the aggregated behavior and results.

The framework included 3 stakeholders in the design process (pedagogical, game design and institutional experts). It was suggested that including end-users and other stakeholders as co-designers in the design process would enable the design of more effective games, and increase buy-in, by ensuring that stakeholder perspectives and requirements are included in the design. The concept of co-design in serious games is also strongly advocated in literature (Braad, Žavcer, \& Sandovar, 2016).

These lessons and unexplored benefits were used to define an initial set of requirements for the revised framework:

- it should enable bidirectional learning where various stakeholders can learn from the game and the aggregated results and interactions.

- it should enable parameterized learning, where learning parameters are dynamically adapted to enable effective learning (enabled by the bidirectional learning capability).

- it should incorporate stakeholders and end-users as co-designers to increase effectiveness and buy-in.

\section{Extension of the Framework}

The framework was extended to incorporate the above-mentioned aspects and simultaneously applied in defining initial design concepts for a new case study to test its usability. In doing so, it was established that the framework lacked flexibility. Although not impossible, it was difficult to incorporate new functionality or concepts without having to rework large portions of the existing framework.

Furthermore, although it had previously been successfully applied in a case study, the framework provided no means to benefit from previous experiences. The new case study required the completion of all the same steps that the first case study did since it did not cater for any form of reusability. Various authors also emphasize the need for a design model to enable reusability, so that serious 
games may be developed more easily, faster and more cost-effectively (Carvalho, 2016; van der Vegt, Westera, Nyamsuren, Georgiev, \& Ortiz, 2016).

Finally, the rather linear structure of the framework was limiting and not practical in contexts where the game requirements and design may change throughout the design process, or where some aspects of the design process may be more/less important, since design iterations are only considered after completing all previous steps. Dynamic adaptation to specific contexts is required. This would also cater for instances where new contextual nuances may become known during the design process, resulting in changes to requirements from the various stakeholders.

Subsequently, four further requirements were defined for the revised framework:

- it should be flexible.

- it should enable the user to benefit from previous experiences and applications.

- it should cater for reusability.

- it should be less linear and allow the configuration of modules according to the priorities and scope of the scenario.

It was established that a more abstract structure, in the form of an architecture, in which the important functionalities are included, without dictating exact steps to follow, would provide for an improved and more flexible structure for serious game design and enable the fulfilment of the abovementioned requirements. Architectures were subsequently considered as an improved solution path.

\section{Architectures For Serious Game Design}

An architecture is an abstract representation of a system, describing the major functions and components, their structure and how they interact with each other (Bass, Clements, \& Kazman, 2013; Carvalho, 2016; University of Houston, n.d.). Its abstraction reduces the complexity to be dealt with at a given time and enables the communication and coordination between its functions and components in a structured manner. By depicting functionality only, without focusing on how these functions are fulfilled, an architecture provides inherent flexibility and support interchangeability/modularity.

\section{Goals and Benefits of Architectures}

The primary goal of an architecture is "to identify requirements that affect the structure of the application" (University of Houston, n.d.). Other goals include:

- $\quad$ exposing the system's structure, whilst hiding its implementation details.

- realizing case studies and scenarios.

- addressing the requirements of various stakeholders.

- improving the quality and functionality offered by the system.

Martínez-Fernández et al. (2013) identify the main benefits of architectures as higher flexibility, the creation of a knowledge repository enabling knowledge transfer, reduced risk through prequalified and proven elements and the interoperability of different systems. Finally, through systematic reuse of common functionalities and component configurations, a reduced time-to-market and cost of the generated system is implied.

\section{Existing Architectures for (Serious) Game Design}

Various architectures exist for adaptive learning game design. Examples include Monterrat et al.'s (2014) architecture for adaptive gamification systems, and the architecture of adaptive educational hypermedia systems proposed by Karampiperis and Sampson (2005). Bellotti et al. (2009) propose an architecture to increase the efficiency and flexibility of game design by decoupling the content 
from the underlying game and its mechanics. BinSubaih and Maddock (2007) propose an architecture that aims to enable game portability across various game engines. Van der Wegt et al. (2016) suggest that to enable easier, faster and cost-effective serious game development, architectures should enable and emphasize reusability and propose an architecture for reusable game components within a niche of pedagogical functionality. Other architectures such as those presented by Folmer (2007) and Furtado et al. (2011) address aspects of reusability between component layers and game subdomains respectively. Whilst many architectures exist, various shortcomings are identified that would, without being addressed, limit the fulfilment of the requirements defined in Section 3.

\section{Shortcomings of Existing Architectures}

Most architectures focus on certain game design aspects or sub-domains only, without providing a holistic architecture of the full game design landscape. Those that do encapsulate the full process, such as that proposed by Folmer (2007), focus foremost on specific functionalities, in this case, reusability.

Although some address aspects of flexibility, the application thereof is limited to the flexible design of the resulting game. None have been found where the architecture itself is flexible where new tools or functionalities can be added as they become available or needed.

A study by Angelov and Hilliard (2014) identified stakeholder management as a leading problem in architecture design and usage. Stakeholders are often poorly identified, and their involvement is limited. As a result, the value for the stakeholders remains unclear and the resulting architecture is ineffective.

\section{Derived Design Brief For An Improved Architecture}

To effectively and efficiently exploit the many advantages of serious games, a structured approach is required for the design of these games, whilst addressing the various limitations discussed. Building on the learnings taken from the initial framework (see Section 3) and literature, an architecture is developed which aims is to support the structured creation of serious games for specific scenarios in a faster, more effective and efficient manner. The following sections discuss the main requirements for the architecture. Since the architecture is dynamic, with the development following a researchby-design approach, this is not an exhaustive list, but rather covers the aspects that are critical to the architecture and constitute its unique contributions.

\section{Bidirectional Learning}

As mentioned previously, bidirectional learning was one of the primary opportunities identified to enable faster, more effective and more efficient serious game design. By incorporating various stakeholders in the design process, clearly identifying and addressing their requirements and incorporating their perspectives throughout the process, the architecture would enable different forms of learning to take place in the various stakeholders, including but not limited to the learner, education provider, real-world environment portrayed in the game and sponsor of the learning or training instance.

The learners would learn from gameplay, whilst the game and other stakeholders would learn from the aggregated behaviors and results. The education provider could, for example, learn about ways in which the content could be improved, whilst the sponsor could learn about the different approaches followed by learners, and the real-world environment could be improved based on suggestions or interactions from gameplay. Depending on the scenario for which the game is being designed, some of these learning perspectives may be more, or less important.

\section{Flexibility}

Technologies, tools, methods and functionalities in the serious gaming and learning environments are very dynamic. As such, it is not possible to guarantee a single set of available tools that will be effective in every scenario, nor will there be a single correct set for a specific scenario, but rather, 
multiple possible solutions. The architecture should therefore be dynamic and flexible, allowing for the addition, removal and adjustment of functionalities, components and features, as newer or better ones become known. The resulting games created using the architecture should be flexible and reconfigurable, in anticipation for unforeseen functionalities.

\section{Modular Structure}

Although the architecture aims to provide a holistic view of the design process, not all functionalities and components are needed, or equally important in all scenarios. The architecture should be modular in structure, providing the flexibility for the resulting games and architecture itself to be configured, tailored, adjusted and improved as needed. Since the aim of the architecture is to create serious games more effectively and efficiently, it should rely on existing, proven functionalities and tools, rather than focusing on full new development in every aspect. By enabling the connection to existing models, tools and architectures, it would allow the reliance on previously tested and proven components.

\section{Reusability and Configurability}

As mentioned previously, enabling and emphasizing reusability further enables easier, faster and costeffective game development. The modular structure of the architecture would enable the incorporation of mechanisms such as reusability to further enable more effective and efficient game design. Individual modules are seen as functional components that can be reused in multiple environments, increasing the efficiency of the game design. Since reuse implies that these components have been implemented, tested and possibly improved in previous settings, it would enable more effective game design. These reusable modules and components, as well as other components, may need to be configured in a different manner to be effective in another scenario. As such, the architecture should be configurable, allowing the configuration or stacking of parts according to the specific context in a structured and guided way.

\section{Parameterized Learning}

The architecture should enable the configuration and adjustment of learning parameters in response to learner interactions and results. Not only would learners be able to learn more effectively and efficiently with parameters adjusted to suit to their preferences, abilities and interactions but based on the aggregated behaviors and results of learners, the available learning trajectories can be better targeted and adjusted. This is a form of bidirectional learning, where the individual learners' learning is affected, but the game and other stakeholders such as content providers can learn from, and be improved by, the aggregated results as well.

\section{Contextualized Adaptation}

The architecture should enable contextualized adaptation of learning material and experiences based on aggregated learner behavior and results in learning trajectories. This would result in improved, parameterized, adaptive games for more effective and efficient learning. In addition, it would lead to increased understanding and knowledge from the perspective of the content provider, a further example of bidirectional learning. The architecture should also provide the constructs to facilitate the game designer in converting the functionalities (the 'what') into the execution thereof (the 'how') that will be effective in the specific context.

\section{A Proposed Architecture For Bidirectional Learning Games}

Based on the design brief presented, an architecture for creating bidirectional learning games is established. The architecture provides structured design support for creating serious games for specific scenarios in a faster, more effective and more efficient manner. By incorporating various stakeholders as co-designers, it provides transparency of the design process and the resulting game 
for the stakeholders. As the architecture acts as a kind of platform for game development, it allows the designers to prioritize functionality for varying scenarios, to configure and tailor the resulting game as desired, while exercising their own creativity. In other words, the architecture allows designers to better and more deliberately explore and exploit the solution space available. Furthermore, by placing the focus on the functional components and their interrelations, the architecture allows the designers to understand, and better anticipate the potential impact on the game design and other functions should priorities change. Where new or alternative tools or approaches are needed, the architecture can be extended or cater for their use to include or connect to them.

\section{Architecture Design Approach}

The architecture development follows a research-by-design approach, whereby the architecture is developed whilst simultaneously being applied in case studies. Design iterations of the architecture are infused by experiences in the various case studies. As indicated in Figure 2, the architecture is never considered exhaustive or complete, but rather, is continuously iterated on as new functionalities and/or experiences become available. The architecture aims to improve the efficiency of serious game design, and due to its flexible, modular structure, and the continuous incorporation of experiences from case studies, it is anticipated that the design iterations will converge to a point where the architecture can be considered sufficiently complete and applicable, until such a point that a new considerable delta is identified, and further iterations are triggered.

The architecture enables the development of flexible games that can be configured, adjusted, tailored and improved as needed. With each iterative improvement of the architecture, these improvements, in turn, lead to improved configurations and tailoring of the game. Furthermore, by enabling reusability, the architecture enables faster, more efficient and more effective development with each application.

Two vertical lines are depicted in the approach, namely the minimum viable product (MVP) and sufficiency criteria. An MVP is a first version of a product that meets the minimum requirements or has sufficient features to satisfy early adopters (Techopedia, 2020). The case study or game being developed will have a set of requirements from the various stakeholders, divided into different priority levels. A minimum acceptable set of requirements will be defined for the game, constituting the MVP. Reaching that MVP state will require one or more development cycles, forming the first version of the game that could be released. The fulfilment of the minimum requirements is determined by the respective stakeholders and is therefore dependent on the application context. The sufficiency criteria are defined as the point at which development can be ceased. These criteria may vary depending on the context and could be when the current set of requirements is fulfilled, or when the changes between subsequent versions becomes negligible and converge.

\section{Overview of the Proposed Architecture}

The architecture, depicted in Figure 3, consists of 19 interconnected functional components. It covers the design and development of the game, as well as the execution thereof (i.e., gameplay). The functions may be fulfilled by a person or software (or a combination thereof) depending on the given scenario. Each function is briefly described in this section, with prioritized functions described in further detail in the sections that follow. 
Figure 2. Research-by-design approach followed in the architecture design

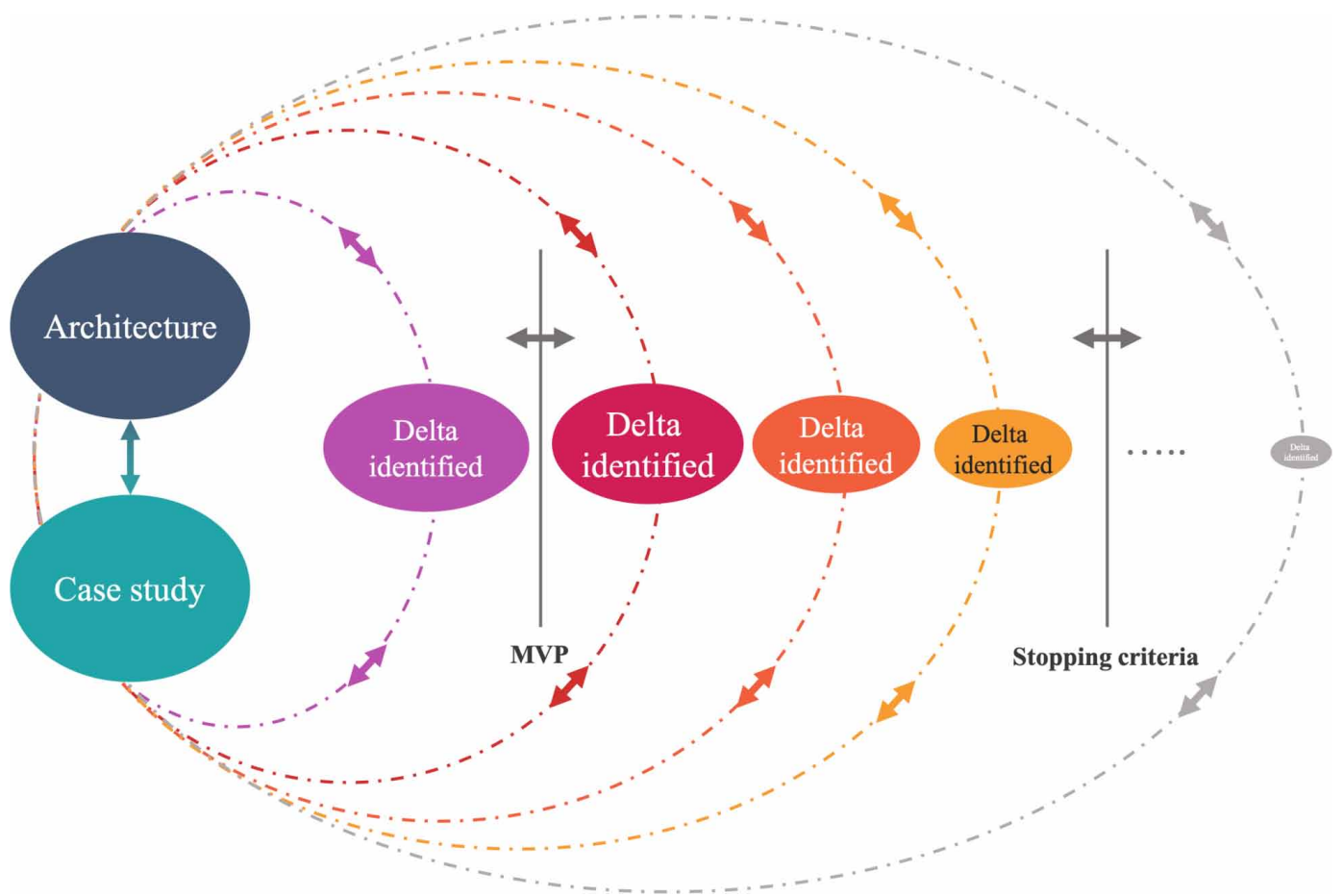

Figure 3. An architecture for bidirectional learning games

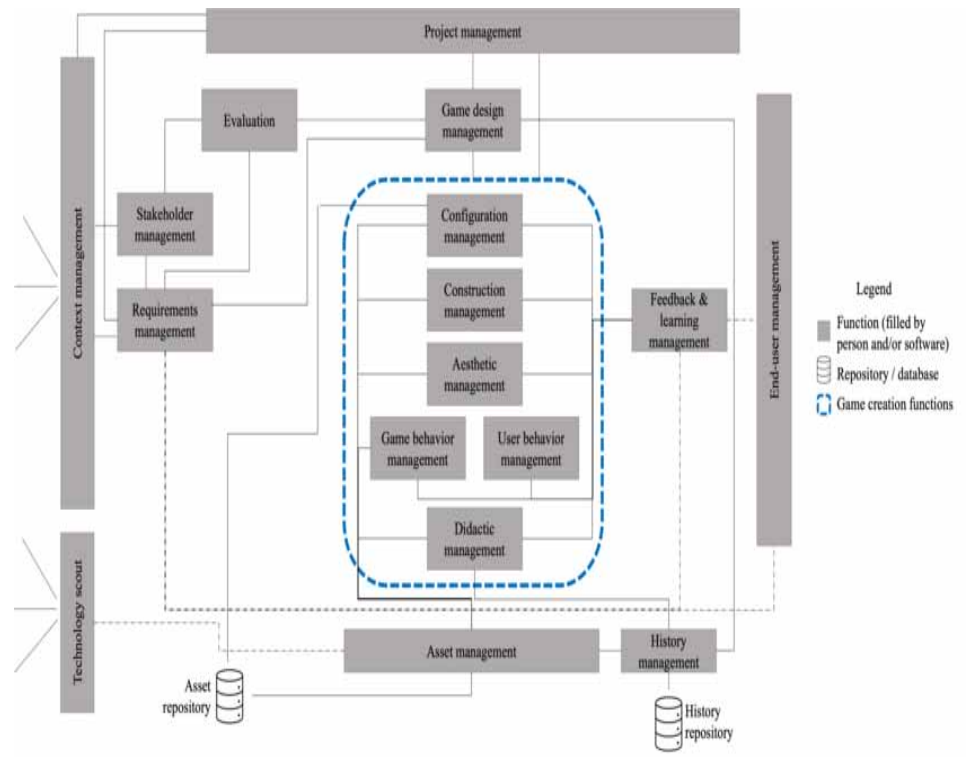


In the center of the architecture, the grouping of functions represents the game creation, which is connected to the context and stakeholders on the left, and the end-user on the right. The context is continuously scouted to enable decision-making in other functions, influencing the requirements and project management aspects. The requirements function handles the measurement and adaptation of requirements. Stakeholder management includes the alignment of the various stakeholders, their requirements, expectations, and involvement in the game design process. The evaluation function evaluates the suitability of the game design and the fulfilment of requirements. The game design function guides the design from requirements to design brief to implementation/delivery. The project management function guides stakeholders through the architecture and design process, from idea to game, considering various constraints including time and budget.

As part of the game creation group, the configuration function assembles/configures existing blocks of functionality (contained in the asset repository) to bring the game to the user. Where new components or blocks of functionality are required, these are created by the construction function. Aesthetic management handles the aesthetic facets of the game, including the visual design/styling and audio. The behavior built into the game is handled by the game behavior function, whilst the user behavior function captures the actual behavior exhibited by the user(s) for comparison to the intended behavior. The didactic function manages the educational content and learning approach, as the main carrier of the intended capacity of the game.

All previously constructed and/or acquired tools, components and functionalities are available for reuse in the asset repository, along with any components and the subsequent experiences of components that are no longer in use. The asset management function considers the available building blocks and components, combined with previous experiences to enable the reusability of components by the configuration function and triggers the construction of new components when required. Versioning and history are maintained for reference by the history functions. The technology scout continuously scouts for new technologies and tools available in the market and informs asset management when new ones become available.

The game is delivered to the end-user(s), with learnings/outputs contextualized and presented to individual users by the end-user management function. This function also manages the user interactions and feedback which is closely related to the feedback and learning function, which in turn captures feedback and learning to enable adaptation and decision-making in other functions of the architecture.

\section{Interactions and Relations Between Architecture Functions}

The architecture focuses on the functionalities of the game design and the interrelations between them. These relations can be unidirectional or bidirectional. In bidirectional connections between, for example, the stakeholder, requirements and evaluation functions, the functions influence each other. The stakeholder function orchestrates the alignment of stakeholders, their requirements and expectations. This, together with the various sets of requirements handled by the requirements function, influences what needs to be evaluated in order to meet their expectations and requirements. Based on the feedback/outcomes of the evaluation, the stakeholders' expectations need to be managed and requirements may need adjustment. All relations can be bidirectional, although some may be less obvious, depending on the context and application. The architecture's flexible structure provides the capacity of changing relations to be unidirectional or bidirectional where needed. The directional relations between functionalities and their importance are interpreted and prioritized by the designer(s) as needed, since this is dependent on the context and requirements of the specific scenario. In addition to the connections/relations between functions, the architecture also enables the connection to existing modules and tools.

\section{Connection to Existing Modules from the Architecture}

The architecture focuses on what the functionalities are and the way in which they are interrelated, without focusing on how they should be executed. This allows for user creativity in the prioritization 
and execution of functions. It also enables the connection to existing models, tools and architectures where they are able to provide the necessary functionality. As an example, the RAGE architecture for reusable components (van der Vegt, Westera, Nyamsuren, Georgiev, \& Ortiz, 2016) could be used in the asset management function. The RAGE architecture was developed with the intention of enabling the faster, easier and more cost-effective development of serious games. By emphasizing interoperability and portability, the architecture supports the development of reusable components for serious games. The architecture presented in this paper allows the user to connect to existing architectures or tools where suitable.

\section{Prioritized Functions of the Architecture}

Whilst some functions in the overall architecture may be well-known (e.g., project management) and others can be fulfilled by existing models (e.g., asset management), others are vital to the faster, more effective and more efficient serious game development and contribute to the identified gaps in literature. As the development of the architecture is driven by a research-by-design approach, is it not predeterminable what functions need to take precedence, given that they are iteratively addressed in the architecture development. However, there are several functions that clearly require consideration from the start; these functions are described in further detail.

\section{Stakeholder Management}

As mentioned previously, one of the primary problems in architecture design is stakeholder management. Various experts agree that involving stakeholders as co-designers provides clarity of purpose, increases the engagement, trust and buy-in of stakeholders, and ensures the effective development of a game that meets the needs of the different stakeholders and is well-suited for its audience (Brandt, 2006; Khaled \& Vasalou, 2014; Melonio, 2013; Plumettaz-Sieber, Hulaas, Sanchez, \& Jaccard, 2019; WACOSS, 2016). Figure 4 depicts an illustration of stakeholders to be included as co-designers, representing various spheres of influence. Some have an impact on the game design (varying from direct control to indirect influence), whilst others are merely impacted by the game and its results. Direct control is exhibited by the end-user/learner, whilst stakeholders such as the content provider, developer and facilitator, sponsor and system have a lower level of control over the game but still exhibit a direct influence. The prioritization of stakeholders and the decision of which to include as co-designers is at the discretion of the designer and is largely dependent on the given context (hence the connection between the context and stakeholder functions). Stakeholders

Figure 4. Stakeholders clustered by spheres of influence

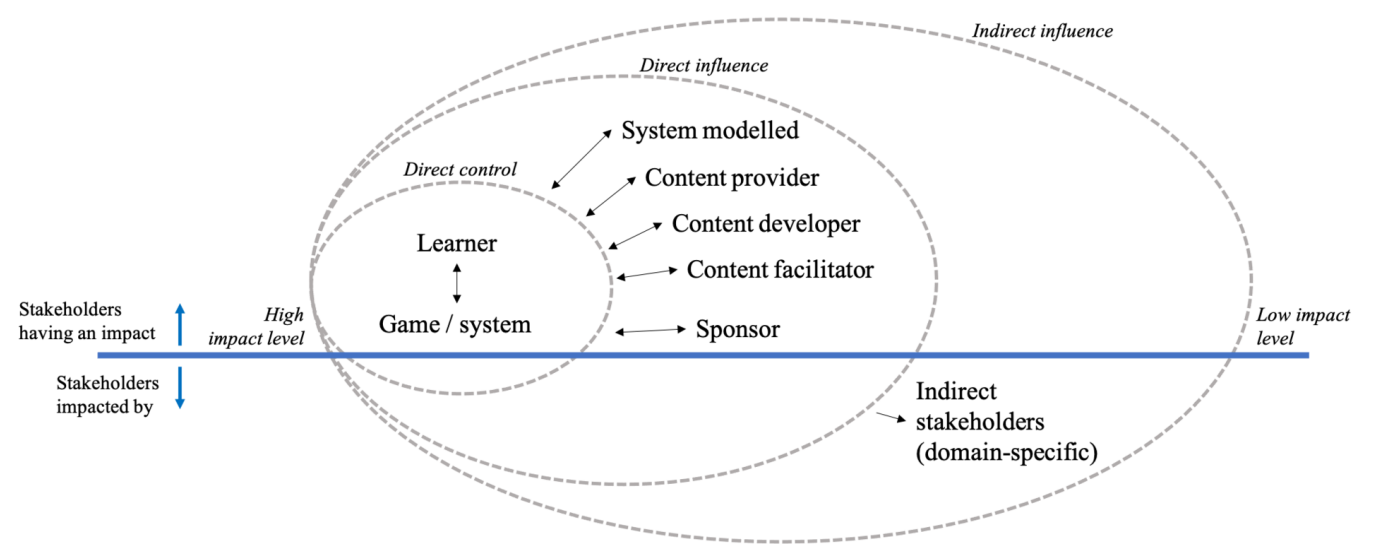


can also be added or deactivated as needed or moved across spheres depending on the scenario and/ or requirements.

\section{Requirements Management}

The requirement's function is closely related to stakeholder management as this function handles the definition and alignment of the various requirements that the stakeholders may have. Although the requirements are usually defined as an initial set for the game design brief, they are dynamic. The context of the game is dynamic and may result in adjusted or even new requirements emerging in later stages of the design process. The requirements function handles the incorporation of these requirements. Based on the feedback from the evaluation function, or learnings from the design or game itself, stakeholders may adjust their existing requirements.

\section{Feedback and Learning}

The game is developed with the intention of improving the quality, effectiveness and efficiency of learning. To enable this, user experiences are captured, aggregated and analyzed in the feedback and learning function. The resulting feedback and learnings are used to inform adaptation and decisionmaking in the various connected functions of the architecture. For example, if users are given a choice between three options in a specific game scenario, and all users select the same option every time, it may be an indication that this is always the best option. The game could be adapted to use this option by default, and rather provide the user with more choices at other points in the game where they are possibly more useful.

\section{Behavior Management}

Game elements and mechanics can trigger or encourage specific user behaviors in a game. When introduced strategically, these elements and mechanics, and their resulting behaviors, can contribute toward more effective and efficient learning in the game. The game behavior function manages the behaviors built into the game. The actual behavior exhibited by user(s) is captured by the user behavior function and compared to the intended behavior. Deviances are analyzed and may trigger an adaptation of the behavioral models and strategies applied.

\section{Didactic Management}

As mentioned previously, different users have different motivators, learning styles, preferences, and approach and play a game differently (Molin, 2017). To be effective, the various individual traits and preferences should be considered and used to provide a highly personalized experience (Akbulut \& Cardak, 2012; All, Castellar, \& Van Looy, 2016; Ferro, Walz, \& Greuter, 2013; Gofron, 2014; González, Toledo, \& Muńoz, 2016; Göbel, Wendel, Ritter, \& Steinmetz, 2010). The didactic function focuses on the personalization and adaptation of learning content and approaches to the preferences, abilities and interactions of the user(s). This portrays the bidirectional learning aspect, where the individual learners learn from the game, but the game also learns from its users and adapts various parameters accordingly.

\section{Applying the Architecture}

The architecture aims to provide support in the design of serious games for specific scenarios, without dictating exactly how it should be done. The users of the architecture can prioritize functionalities based on the context the game is being designed for, using the architecture to better understand the relations between the various functionalities. The architecture is applicable across the entire game design and development process and caters for dynamic priorities and requirements. If/when priorities or requirements change, the user can use the architecture to understand what the impact on other areas and functionalities might be by considering the interrelations between functions. Functionalities may also be prioritized and deprioritized dynamically, as required for the specific scenario. Users can 
use existing tools and components already contained in their asset repository or add to it as needed. They can also connect directly to other existing models and tools for relevant functions. In Section 7 , the pilot applications are discussed.

\section{Validation}

The primary aim of the architecture is to provide users with structured support in creating serious games for specific scenarios in a faster, more effective and efficient manner. The underpinned requirements are set out in the design brief in Section 5. To validate the architecture, the usability of the architecture, as well as the existence of these underpinned requirements, must be demonstrated.

\section{Validation Strategy}

Following a research-by-design approach, the architecture is developed and validated through its iterative application in case studies. In this, the focus is on how the use of the architecture drives the game development with regards to its usability, and the existence of the underpinned requirements such as bidirectional learning, flexibility, modular structure, reusability and configurability, parameterized learning and contextual adaptation. Each application may utilize and prioritize different combinations of functionalities depending on the context and objectives, demonstrating the configurability of the architecture and the ability to interchange modules and functionality of the architecture, enabling the creation of serious games in a faster, more effective and efficient manner. Various stakeholders are included as co-designers in the case studies. Through workshops, discussions and interviews, their various requirements, perspectives and feedback are captured and incorporated into the case studies and subsequent design iterations of the architecture. Learnings, opportunities and possible shortcomings identified in each application are infused as enhancements into the next design iteration of the architecture. A pilot application is described, providing specific examples of various components and aspects demonstrating various functionalities of the architecture.

\section{Case study 1: A Design Concept for Diagnostic Performance Improvement Training}

In an initial case study, the architecture was applied focusing on defining a design brief and concept of a serious game for a performance improvement training program for diagnostic laboratories. The architecture functionalities prioritized included the stakeholder, requirements, evaluation and game design functionalities. Various brainstorming sessions were held with the training content providers for initial idea generation and scoping, as well as the initial definition of stakeholders and requirements. The anticipated stakeholders were classified by spheres of influence and some initial ideas and concepts for a possible game scenario were defined.

Using these initial requirements and concepts as input, a focus group workshop was held with a group of 10 participants, each representing one or more of the direct stakeholders identified, namely the learner (9), the game (2), content provider (2), laboratory (4) and sponsor (1). The workshop was aimed at refining the anticipated stakeholder spheres and requirements, prioritizing these requirements, defining initial game concepts and evaluating their feasibility in fulfilling the requirements. Sample scenarios and storyboards were defined and prototyped to further discuss and better understand the potential of such a game. In addition to the workshop, three diagnostic laboratories in the Chicago area were visited to gain a better understanding of laboratory scenarios. Various lab processes were observed and evaluated to gain critical insights to be incorporated into the game.

Through the exposure to the architecture, stakeholders were able to understand the perspectives of others in the group, make improvements, and learn from the process itself. The resulting, prioritized requirements of these initial interactions were compiled as a design brief and given to a designer for the second case study. 


\section{Takeaways and Results}

The overall feedback received from the various stakeholders was positive, with stakeholders recognizing the potential of such a game concept and the architecture for creating it. Various takeaways from the interactions were incorporated into the architecture in subsequent design iterations. One such example is the importance of enabling the dynamic (re)prioritization of requirements. In this case study, anticipated requirements were defined for each stakeholder and were subsequently confirmed and expanded on in the workshop. Through the discussions around the different stakeholder perspectives, a sponsor requirement initially classified as a showstopper was deprioritized to a nice-to-have and replaced with a requirement that emerged through the discussions. This demonstrated the flexibility of the architecture in enabling the dynamic prioritization of requirements, as well as the value in the learn-by-doing approach it emphasizes.

\section{Case study 2: A Prototype for Diagnostic Performance Improvement Training}

In the second case study, the emphasis was on demonstrating the usability of the architecture from a designer's perspective. Using the design brief, initial ideas and prototype from the first case study, the designer used the architecture to prioritize functionalities and their interrelations throughout the game development process.

The developed game is situated in a histology laboratory, in which the overall aim is to identify opportunities to improve the laboratory performance. The game incorporates the perspectives of the various stakeholders and can be played by different stakeholders, collectively referred to as actors.

The architecture aims to provide a modular, flexible structure in which relevant functionalities can be prioritized for a given scenario. In this case study, the prioritized functionalities include the stakeholder, didactic, feedback and learning, game and user behavior functions as well as the asset, configuration and construction functions. The aesthetic function was initially deprioritized but was later incorporated to introduce multiple aesthetic variants of the game to cater for different player experience levels, further demonstrating the architecture's flexibility. New or inexperienced players are introduced to the game in a simplified, white laboratory where important and interactable game objects, as well as learning materials and lessons are easily identifiable (Figure 5[REMOVED REF FIELD]a). As the actor gains experience, the game environment becomes more realistic (Figure 5b), making important objects more difficult to find, bringing the experience closer to the real-world scenario, and increasing the emersion in, and complexity of, the learning scenario.

Figure 5. Aesthetic variations of the game environment portraying (a) a basic variation and (b) a realistic variation

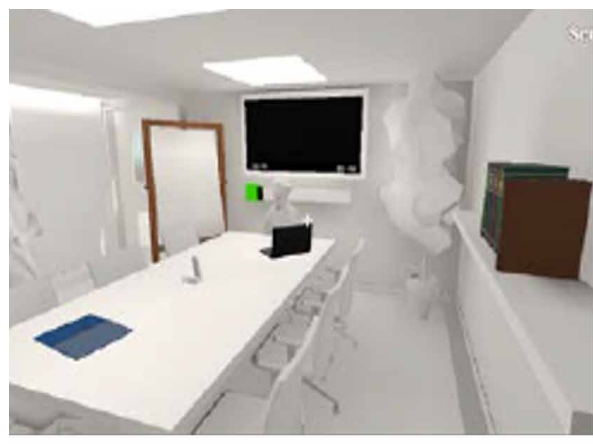

$a$

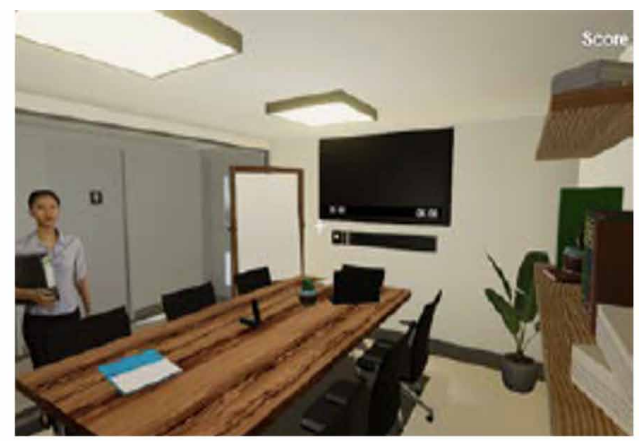

$b$ 
Building on the modular structure, the architecture enables reusability of existing components and configurability to improve the effectiveness and efficiency of the design process and resulting game. The configuration and asset management functions enable the repurposing, adapting, and reuse of previously created components, while the construction function enables the construction of new components as needed. The designer was able to repurpose and adapt components from previously developed prototypes and games, demonstrating the concept of reusability in the architecture. A dialogue system (Figure 6a) was adapted from a previous game to be more modular. Various components are reused and configured in different ways in multiple aspects of the game. The script controlling the reading resources (Figure 6b), was adapted and reused for the notebook component where actors can capture important information and notes (Figure 6c).

Figure 6 . Components repurposed by the configuration function including the (a) dialogue system, (b) bookshelf with books, and (c) notebook

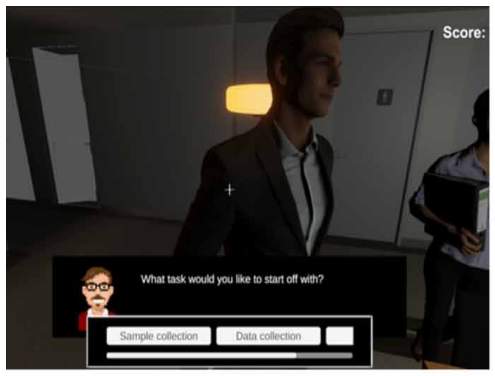

$a$

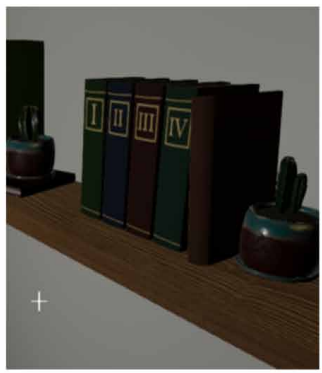

$b$

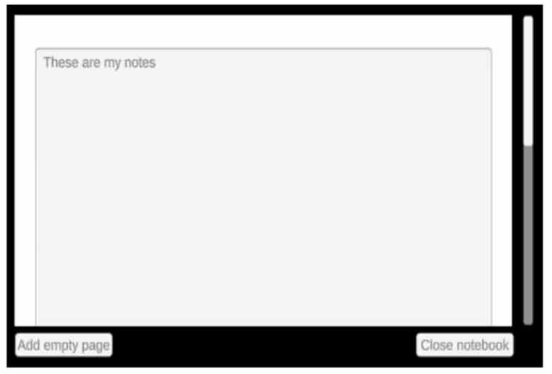

$c$

An important requirement for the architecture is the emphasis on bidirectional learning, where different forms of learning are enabled for various stakeholders. A useful learning outcome for the content provider is the identification of improvement opportunities for the learning material. Quiz response times may indicate concepts that learners struggle with where content should be adapted. Additional reading material is available, and actors can capture important information in notebooks. An aggregated analysis of the content added to notebooks may suggest important concepts that are not sufficiently understood without further reading and should be incorporated into the game and content directly. In contrast, books that are never used may be deemed unimportant or readily understood and could be removed.

Learning outcomes for the laboratory include layout or workflow improvements. In the prototype, actors can select objects in the laboratory and add notes or suggestions (Figure 7a). An analysis of these notes may indicate a suboptimal layout that could be improved by following the suggestions made. Actors are sent to specific locations in the laboratory for certain tasks. The routes followed are tracked and captured and could be plotted on a heatmap to indicate improvement opportunities for the laboratory layout. These examples demonstrate bidirectional learning on a meta-level, where the actor is learning about laboratory improvement practices, whilst other stakeholders simultaneously learn from the aggregated behaviors of actors. Taking the heatmap example: Actors are learning about the laboratory and performance improvement, whilst the heatmap of routes taken tells the game designer(s) where actors spend most of their time and may suggest layout improvements for the laboratory.

The game itself can also be improved through bidirectional learning elements. A whiteboard (Figure $7 \mathrm{~b}$ ) displays common questions and answers. Actors can ask additional questions, which, 
if asked frequently or deemed important, can be answered in the backend database and will then be updated on the whiteboard. When the actor completes or ends the game, they are approached by the laboratory manager and asked to provide their feedback on the experience (Figure 7c). The aggregated feedback can be used to further improve the game.

The architecture also aims to enable parameterized learning and contextualized adaptation of learning materials and experiences. Quiz results have banded responses, where relevant resources are provided if an actor's score falls within a given band. As the actor gains experience, the laboratory design becomes more realistic, adding to the complexity of the game for that actor. These examples demonstrate the adaptation of learning parameters to an actor's interactions, abilities and results. Examples of contextualized adaptation include the resources described previously. If many actors are using specific resources, these should be incorporated directly into the game. In contrast, resources that are never used should be removed and replaced by other, potentially more suitable resources.

Figure 7. Bidirectional learning components including the (a) placing of notes, (b) FAQ whiteboard, and (c) exit interview

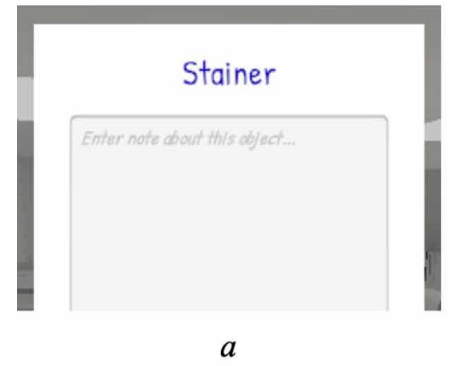

$a$

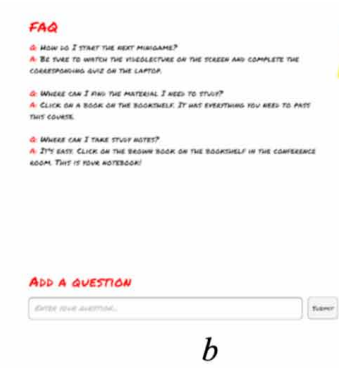

$b$

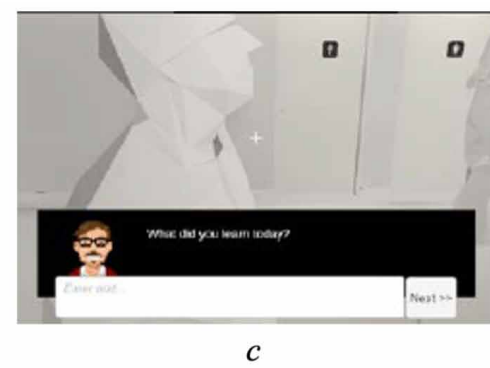

\section{Takeaways and Results}

This case study demonstrated the architecture's flexibility through the ability to prioritize and deprioritize functionalities dynamically throughout the development process. The aesthetic function was initially deprioritized in the prototype development but was later prioritized and incorporated to enable the adaptation of aesthetic realism in response to player experience and performance. The emphasis on reusability in the architecture was also demonstrated through the reuse of various game components in different aspects of the game, as well as the repurposing of features from previously developed games in the asset repository. Throughout the application, various improvement opportunities were identified and incorporated into subsequent design iterations. One such learning was the incorporation of an identity management function within the stakeholder function of the architecture, to identify the different perspectives and preferences of actors more explicitly in the game.

Although the developed prototype was not tested through gameplay, it was presented to representatives of the various stakeholders for feedback. Stakeholders were able to identify and appreciate the learning opportunities the game presents for the different stakeholders. The content provider especially realized the value in analyzing the aggregated behaviors and results of learners to identify improvement opportunities for the training content and delivery.

\section{Implementation Results and Impact}

The architecture was successfully applied in two case studies, demonstrating its usability. In each case study, different functionalities were prioritized as relevant for the specific context and application phase, demonstrating the flexible, modular nature of the architecture. The case studies also 
incorporated different stakeholders, to varying degrees as required by the scenario. The reusability and configurability of the architecture was demonstrated by the repurposing and reuse of components in different game aspects, as well as across case studies. Learning parameters are configured and adjusted in response to user interactions and results, demonstrating the parameterized learning capability of the architecture.

Through the architecture application in these two case studies, various improvement opportunities were identified, which will be incorporated into the next version of the architecture. A stronger emphasis is required on identifying the different perspectives and preferences of actors in the game, to enable more effective adaptation of content and mechanisms. Identity management should therefore be included within the stakeholder function. A need to emphasize the holistic planning and robustness of the game, communication between the various functions, as well as playtesting was identified. A progress management function will be added to the architecture which encompasses these improvements, allowing designers to constantly evaluate the current state of the game against the planned end goal. A final suggestion was made to distinguish between data captured from past versions of the game, and the evolution of the game itself in the history functions. The history management function will be split to accommodate this improvement, with one part focusing on data collection to allow for effective comparison between data captured from different versions, and the second on the design rationale and changes behind different versions of the game itself.

The overall feedback from users was positive, with the designer of the second case study stating that "throughout the development process, the architecture enabled the more deliberate exploration of the available serious gaming solution space. Moreover, its modular nature and focus on reusability enables the effective and efficient realization of serious games".

Following a research-by-design approach, the application of the architecture enables the identification of enhancements which are infused as learnings into the next version of the architecture, demonstrating the flexibility of the architecture. The architecture is applied in further case studies following the same approach.

\section{Conclusion}

Dynamic personalization of learning trajectories that integrate different perspectives and variable scenarios is a purposeful and viable way to improve the quality, effectiveness and efficiency of training and education. Serious games offer a designated platform for this. An architecture is presented to support the structured creation of such games for specific scenarios in a faster, more effective and efficient manner. The architecture's modular structure provides the flexibility for the resulting games and the architecture itself to be configured, tailored, adjusted and improved as needed. It enables the connection to existing models, tools and architectures in the relevant functions of the architecture. Mechanisms such as reusability further contribute towards more effective and efficient serious game design. Moreover, as the architecture inherently capacitates bidirectional learning, the contextualized adaptation of learning material and experiences based on the aggregation of learner behavior/results in learning trajectories is enabled. This ultimately results not only in improved, parameterized, adaptive games for more effective and efficient learning, but also in increased understanding and knowledge for the provider of the education.

Initial validation in the form of expert interviews yielded positive results. To demonstrate the usability of the architecture, it is applied in two case studies. Following a research-by-design approach, the required changes identified will be incorporated into further iterations of the architecture.

\section{Recommendations}

Whilst the development of the case studies demonstrates the usability and potential of the architecture and resulting game, further testing and evaluation by application (case studies) is required to fully validate the architecture and its goals. The case studies are not intended to prove completeness or correctness of the architecture, but rather, demonstrate the validity of the architecture and offer further 
enhancements. The modularity of the architecture allows for future configuration, tailoring, adjustments and improvements. If further case studies show a convergence of usability and improvements, it would demonstrate the validity of the approach. Furthermore, although the case studies introduced in this paper focus on performance improvement in diagnostic laboratories, it is suggested that the architecture would also be applicable in other contexts. It is therefore recommended that further case studies in other contexts are developed in order to validate its usability and applicability in other contexts.

\section{ACKNOWLEDGMENT}

The authors would like to thank the Power of Process (Pty) Ltd (Power of Process, 2020) for providing their training materials as a basis for the case study and for their participation in the design workshops. 


\section{REFERENCES}

Akbulut, Y., \& Cardak, C. S. (2012). Adaptive educational hypermedia accomodating learning styles: A content analysis of publications from 2000 to 2011. Computers \& Education, 58(1), 835-842. doi:10.1016/j. compedu.2011.10.008

All, A., Castellar, E. P., \& Van Looy, J. (2016). Assessing the effectiveness of digital game-based learning: Best practices. Computers \& Education, 92(1), 90-103. doi:10.1016/j.compedu.2015.10.007

Amory, A., \& Seagram, R. (2003). Educational game models: Conceptualisation and evaluation. South African Journal of Higher Education, 17(2), 206-217.

Angelov, S., \& Hilliard, R. (2014). Towards an improved stakeholder management for software reference architectures. In 8th European Conference on Software Architecture (pp. 90-97). doi:10.1007/978-3-319-099705_9

Bass, L., Clements, P., \& Kazman, R. (2013). Software architecture in practice (3rd ed.). Addison-Wesley Professional.

Bellotti, F., Berta, R., De Gloria, A., \& Primaver, L. (2009). Adaptive experience engine for serious games. IEEE Transactions on Computational Intelligence and AI in Games, 1(4), 264-280. doi:10.1109/TCIAIG.2009.2035923

BinSubaih, A., \& Maddock, S. (2007). G-factor portability in game development using game engines. 3rd International Conference on Games Research and Development, 163-170.

Braad, E., Žavcer, G., \& Sandovar, A. (2016). Processes and models for serious game design and development. In R. Dörner (Ed.), LNCS Entertainment Computing \& Serious Games. Springer. doi:10.1007/978-3-319-46152-6_5

Brandt, E. (2006). Designing Exploratory Design Games: A Framework for Participation in Participatory Design. Participatory Design Conference. doi:10.1145/1147261.1147271

Carvalho, M. B. (2016). Serious Games for Learning: A model and a reference architecture for efficient game development. Technische Universiteit Eindhoven.

Cilliers, S. (2016). The five simple truths about training "the right way". Heavy Chef Review: Education, 4(1), $27-28$

Codish, D., \& Ravid, G. (2014). Personality based gamification - Educational gamification for extroverts and introverts. Proceedings of the 9th CHAIS Conference for the Study of Innovation and Learning Technologies: Learning in the Technological Era, 1, 36-44.

Connoly, T. M., Boyle, E. A., MacArthur, E., Hainey, T., \& Boyle, J. M. (2012). A systematic literature review of empirical evidence on computer games and serious games. Computers \& Education, 59(2), 661-686. doi:10.1016/j.compedu.2012.03.004

Dickey, M. D. (2005). Engaging by design: How engagement strategies in popular computer and video games can inform instructional design. Educational Technology Research and Development, 53(2), 67-83. doi:10.1007/ BF02504866

Ferro, L. S., Walz, S. P., \& Greuter, S. (2013). Towards personalised, gamified systems: an investigation into game design, personality and player typologies. Proceedings of the 9th Australasian Conference on Interactive Entertainment: Matters of Life and Death, 7-13. doi:10.1145/2513002.2513024

Folmer, E. (2007). Component based game development-a solution to escalating costs and expanding deadlines? Component-Based Software Engineering, 66-73.

Furtado, A. W., Santos, A. L., Ramalho, G. L., \& De Almeida, E. S. (2011). Improving digital game development with software product lines. IEEE Software, 28(5), 30-37. doi:10.1109/MS.2011.101

Gee, J. P. (2003). What video games have to teach us about learning and literacy. Computers in Entertainment, 1(1), 20-20. doi:10.1145/950566.950595

Göbel, S., Wendel, V., Ritter, C., \& Steinmetz, R. (2010). Personalised, adaptive digital educational games using narrative game-based learning objects. Entertainment for Education (Edutainment), 438-445. 
Gofron, B. (2014). School in the era of the internet. Educ. Educ., 17(1), 171-180. doi:10.5294/edu.2014.17.1.9

González, C. S., Toledo, P., \& Muńoz, V. (2016). Enhancing the engagement of intelligent tutorial systems through personalization of gamification. International Journal of Engineering Education, 32(1(B)), 532-541.

Hall, N., Watson, M. J., \& Kitching, A. (2017). Serious About Games: Special kinds of video games can revolutionise teaching and learning - and the Western Cape game development industry can deliver them. Serious About Games.

Hamari, J., Shernoff, D. J., Rowe, E., Coller, B., Asbell-Clarke, J., \& Edwards, T. (2016). Challenging games help students learn: An empirical study on engagement, flow and immersion in game-based learning. Computers in Human Behavior, 54(1), 170-179. doi:10.1016/j.chb.2015.07.045

Kalyanakrishnan, S., \& Stone, P. (2011). Characterizing reinforcement learning methods through parameterized learning problems. Machine Learning, 84(1), 205-247. doi:10.1007/s10994-011-5251-x

Karampiperis, P., \& Sampson, D. (2005). Adaptive learning resources sequencing in educational hypermedia systems. Journal of Educational Technology \& Society, 8(4), 128-147.

Khaled, R., \& Vasalou, A. (2014). Bridging serious games and participatory design. International Journal of Child-Computer Interaction, 2(2), 93-100. doi:10.1016/j.jicci.2014.03.001

Marne, B., Wisdom, J., Huynh-Kim-Bang, B., \& Labat, J.-M. (2012). The six facets of serious game design: A methodology enhanced by our design pattern library. In A. Ravenscroft, S. Lindstaedt, C. D. Kloos, \& D. Ández-Leo (Eds.), 21st Century Learning for 21st Century Skills: 7th European Conference of Technology Enhanced Learning (Vol. 7563, pp. 208-221). Springer Berlin Heidelberg. doi:10.1007/978-3-642-33263-0_17

Martínez-Fernández, S., Ayala, C. P., Franch, X., \& Martins Marques, H. (2013). Benefits and drawbacks of reference architectures. 7th European Conference on Software Architecture. doi:10.1007/978-3-642-39031-9_26

Melonio, A. (2013). Game-based Co-design of Games for Learning with Children and Teachers: Research Goals and $a$ Study. CEUR.

Minović, M., \& Milovanović, M. (2013). Real-time learning analytics in educational games. ACM., doi:10.1145/2536536.2536574

Molin, G. (2017). The role of the teacher in game based learning: A review and outlook. Serious Games and Edutainment Applications, 649-674.

Monterrat, B., Desmarais, M., Lavoué, E., \& George, S. (2015). A player model for adaptive gamification in learning environments. AIED, 2015(1), 297-306. doi:10.1007/978-3-319-19773-9_30

Monterrat, B., Lavoué, É., \& George, S. (2014). Toward an adaptive gamification system for learning environments. International Conference on Computer Supported Education, 115-129.

Oblinger, D. G. (2004, May 21). The next generation of educational engagement. Journal of Interactive Media in Education, 8(1), 1-18.

Plumettaz-Sieber, M., Hulaas, J., Sanchez, E., \& Jaccard, D. (2019). Co-design of a serious game for computing education. The Gamification \& Serious Games Symposium 2019 (GSGS'19), Neuchâtel, Switzerland.

Power of Process. (2020). Retrieved March 12, 2021, from https://joinpop.org/

Romero, M., Usart, M., \& Ott, M. (2015). Can serious games contribute to developing and sustaining 21st century skills? Games and Culture, 10(2), 148-177. doi:10.1177/1555412014548919

Shi, L., Christea, A. I., \& Hadzidedic, S. (2014). The critical role of profiles in social E-learning design. In 15th Annual Conference on Information Technology Education (SIGITE 2014) (pp. 71-76). WRAP. doi:10.1145/2656450.2656458

Techopedia. (2020). Retrieved 12 8, 2020, from https://www.techopedia.com/definition/27809/minimum-viableproduct-mvp

University of Houston. (n.d.). College of Science and Engineering: Software Architecture \& Design Introduction. Retrieved 11 15, 2020, from https://sceweb.sce.uhcl.edu/helm/SWEN-SAD/my_files/TableContents/Module-14/ quick_guide.html 
van der Vegt, W., Westera, W., Nyamsuren, E., Georgiev, A., \& Ortiz, I. M. (2016). RAGE Architecture for reusable serious gamng technology components. International Journal of Computer Games Technology, 2016(3), 1-10. doi:10.1155/2016/5680526

von Leipzig, T., von Leipzig, K. H., \& Hummel, V. (2019). 21st Century knowledge dissemination: Adaptive games to address the skills gap. In International Conference on Competitive Manufacturing '19 (pp. 366-374). Stellenbosch: Stellenbosch University.

WACOSS. (2016). The co-design toolkit. WACOSS.

Wright, J. (2015). Personalised learning: An overview. Institute for Teaching and Learning Innovation.

Zhang, X.-Y., Wang, S., \& Yun, X. (2015). Bidirectional Active Learning: A Two-Way Exploration Into Unlabeled and Labeled Data Set. IEEE Transactions on Neural Networks and Learning Systems, 26(12), 3034-3044. doi:10.1109/TNNLS.2015.2401595 PMID:25730833

Tanja von Leipzig holds a B.Eng degree in Industrial Engineering and an M.Sc degree in Operations Management. She is currently completing her PhD in Industrial Engineering at Stellenbosch University, with her specific area of focus and research being that of bidirectional, adaptive games for training and education. She is currently working as a Data Engineer at Spatialedge where she is also involved in the training development for various big data engineering related disciplines.

Eric Lutters was educated as a Mechanical Engineer at the University of Twente, where he continued to work as an assistant professor. Simultaneously, he did his PhD, which he finished in 2001 with the thesis 'Manufacturing integration based on Information Management'. He currently holds a position as an associate professor at the Faculty of Engineering Technology at the University of Twente in the Netherlands. Eric leads a research group that focuses on 'Information driven product development', with special interest in Digital Twinning, Virtual/Augmented reality, synthetic environments and 'what-if'-simulations. Together with his group he is responsible for a wide variety of courses in Mechanical Engineering, Industrial Design Engineering and Industrial Engineering \& Management. Eric is the professor of the MSc. specialisation 'Management of Product Development', in which over 150 students are enrolled to focus on the synthesis between stakeholders, information, processes, perspectives and tools in developments cycles. He has a keen interest in project education, educational innovations and quality assurance in education. Eric is a board member of the Dutch platform for post-academic education in technology and management. Next to his work at the university, he is a scientific board member of the Fraunhofer Project Center at the University of Twente. Eric is a Fellow of the International Academy for Production Engineering (CIRP), where he is also a member of the council. He is the author of a number of textbooks in the field of design and manufacturing and he is associate editor of a number of journals. Additionally, Eric is a Professor Extraordinary at the Department of Industrial Engineering at the Stellenbosch University in South Africa.

Vera Hummel has been a Professor at Reutlingen University, faculty ESB Business School, since 2010. Her research work focuses on the design of work and logistics systems in smart factories with use of innovative technologies, e.g. stationary and mobile human-robot collaboration (HRC) that increase efficiency of production and logistics systems are investigated by using artificial intelligence and sensors as well as on learning factories as innovative methods for education and training. She graduated in 1988 from the University of Applied Science in Konstanz with a degree in Dipl.-Ing (FH) Mechanical Engineering and received her doctorate Doktorlngenieur in 2002 from the Wroclaw University of Technology. In the years 1988-1994 she was working for Mercedes-Benz in Switzerland and South Africa in the engineering departments, from 1994 till 2008 at the Fraunhofer IPA, Stuttgart in the field of enterprise management and the University Stuttgart in versatile factories. Prof. Hummel is currently the head of the Reutlingen Zentrum Industrie 4.0 as well as the initiator and director of the Werk150 of the ESB Business School. She holds the position of Vice Dean Research for the ESB Business School and an extraordinary Professorship at Stellenbosch University, department Industrial Engineering.

Cornè Schutte is the Chairman of the Department of Industrial Engineering, and Professor at the University of Stellenbosch. He is an Honorary Fellow (FSAIIE) and past president of the Southern African Institute for Industrial Engineering (SAIIE), and is a professionally registered engineer. He has an M.Eng in Industrial Engineering from the University of Pretoria, and a PhD in Engineering from the Stellenbosch University. His research includes the application of Industrial Engineering in Southern Africa, Innovation Management, Knowledge Management, and Engineering Management. 